\title{
Matrix elements of heavy-light mesons from a fine lattice
}

\author{
A. Ali Khan*,A. Al-Haydari' ${ }^{a}$, V. M. Braun ${ }^{b}$, S. Collins ${ }^{b}$, M. Göckeler ${ }^{b}$, \\ G. N. Lacagnina ${ }^{c}$, M. Panero ${ }^{b, d}, \mathbf{A}$. Schäfer ${ }^{b}$, and G. Schierholz $\mathbf{z}^{b, e}$ \\ a Department of Physics, Faculty of Science, Taiz University, Taiz, Yemen Republic \\ ${ }^{b}$ Institute for Theoretical Physics, University of Regensburg, 93040 Regensburg, Germany \\ ${ }^{c}$ INFN, Sezione di Milano, 20133 Milano, Italy \\ ${ }^{d}$ Institute for Theoretical Physics, ETH Zürich, 8093 Zürich, Switzerland \\ e Deutsches Elektronen-Synchrotron DESY, 22603 Hamburg, Germany
}

We performed a calculation of matrix elements of heavy mesons on a quenched lattice, generated with Wilson gauge fields at $\beta=6.6$ with a lattice size of $40^{3} \times 80$ and a lattice spacing $a^{-1} \simeq 5$ $\mathrm{GeV}$ determined from the Sommer parameter $r_{0}=0.5 \mathrm{fm}$. We use a non-perturbatively $O(a)$ improved Wilson fermion action and improved currents.

We have calculated the charmonium spectrum as well as form factors of semileptonic decays of pseudoscalar heavy-light mesons containing a $c$ or a $b$ quark to pseudoscalar light mesons through a vector current:

$$
\left\langle P(p)\left|V^{\mu}\right| H\left(p_{H}\right)\right\rangle=\frac{m_{H}^{2}-m_{P}^{2}}{q^{2}} q^{\mu} f_{0}\left(q^{2}\right)+\left(p_{H}^{\mu}+p^{\mu}-\frac{m_{H}^{2}-m_{P}^{2}}{q^{2}} q^{\mu}\right) f_{+}\left(q^{2}\right),
$$

where $p$ and $m_{P}$ are the momentum and the mass of the light meson respectively, and $p_{H}$ and $m_{H}$ the momentum and the mass of the heavy meson respectively. $q=p_{H}-p$ is the momentum transfer. $V^{\mu}$ denotes a local vector current. A comparison with other lattice calculations for the decay $B \rightarrow \pi l v$ is shown in Figure 1 .

For the decay constant of the $J / \psi$ meson we find a preliminary value of $f_{V}=341(10) \mathrm{MeV}$, using the definition

$$
\left\langle 0\left|V_{j}\right| J / \psi\right\rangle=f_{V} m_{J \psi} \varepsilon_{j}(\lambda)
$$

where $\varepsilon_{j}(\lambda)$ is the polarization vector of the $J / \psi$. We obtain the following values for mass splittings of charmonium states: $\Delta M\left(J / \psi-\eta_{c}\right)=74(2) \mathrm{MeV}$ and $\Delta M\left(\chi_{c 1}-J / \psi\right)=394(15)$ $\mathrm{MeV}$, where the errors are only statistical.

8th Conference Quark Confinement and the Hadron Spectrum September 1-6 2008

Mainz, Germany

\footnotetext{
* Speaker.
} 


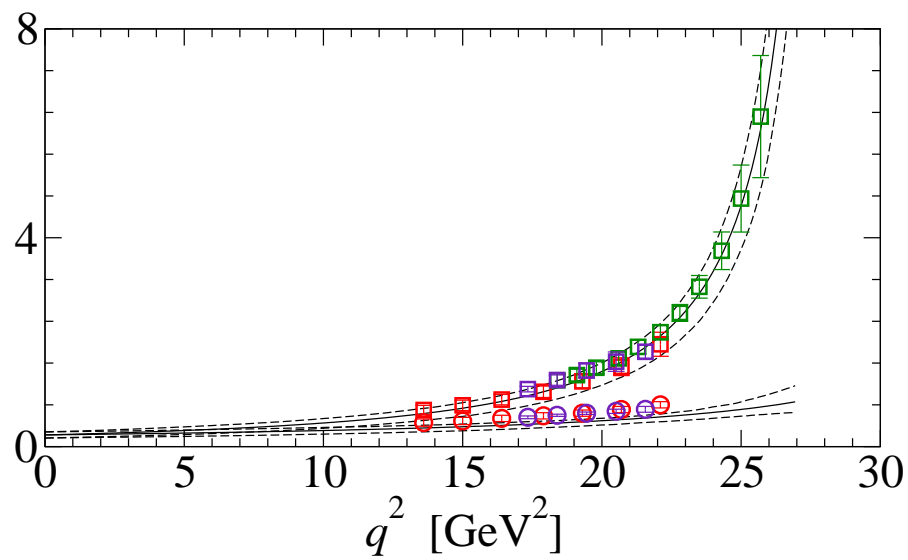

Figure 1: The solid lines denote our results for the form factors $\left(f_{+}\right.$: upper line, $f_{0}$ : lower line $)$ of the decay $B \rightarrow \pi l v$. The dashed lines denote our error bounds. The squares denote $f_{+}$, circles denote $f_{0}$ from other recent lattice calculations (red: quenched, [2], green: $N_{f}=2+1$, [3], magenta: $N_{f}=2+1$, [4])

The details of our calculation of the matrix elements and the results for the form factors at $q^{2}=0$ are given in Ref. [1]. We observe a relatively good agreement of our form factors with other lattice calculations.

Our results for the charmonium spectrum are in very good agreement with a previous quenched calculation at the same lattice spacing [5] and in agreement with a recent calculation with two heavy flavors [6]. Since we work on fine lattices where discretization effects are under good control for charmonia it is also of interest to calculate the charmonium decay constants. Our result for the decay constant of the $J / \psi$ is lower than the result of 399(4) MeV from quenched anisotropic lattices of [7] and of 413(40) $\mathrm{MeV}$ from lattices with $N_{f}=2$ of [8]. The experimental value is 411(7) MeV.

Acknowledgement: We thank J. Simone for useful discussions.

\section{References}

[1] QCDSF collaboration, A. Al-Haydari et al., in preparation.

[2] A. Abada et al., Nucl. Phys. B 619, 565 (2001) [arXiv:hep-lat/0011065].

[3] J. Bailey et al., arXiv:0811.3640 [hep-lat].

[4] E. Dalgic et al., Phys. Rev. D 73 (2006) 074502 [Erratum-ibid. D 75 (2007) 119906] [arXiv:hep-lat/0601021].

[5] S. Choe et al. [QCD-TARO Collaboration], JHEP 0308, 022 (2003) [arXiv:hep-lat/0307004].

[6] C. Ehmann and G. Bali, PoS LAT2007, 094 (2007) [arXiv:0710.0256 [hep-lat]].

[7] J. J. Dudek, R. G. Edwards and D. G. Richards, Phys. Rev. D 73, 074507 (2006) [arXiv:hep-ph/0601137].

[8] P. Dimopoulos, C. McNeile, C. Michael, S. Simula and C. Urbach [ETM Collaboration], arXiv:0810.1220 [hep-lat]. 